APS

physics

This is the accepted manuscript made available via CHORUS, the article has been published as:

van der Waals interaction as a summable asymptotic series John P. Perdew, Adrienn Ruzsinszky, Jianwei Sun, Stephen Glindmeyer, and Gabor I. Csonka

Phys. Rev. A 86, 062714 - Published 26 December 2012 DOI: 10.1103/PhysRevA.86.062714 


\title{
Van der Waals Interaction as a Summable Asymptotic Series
}

John P. Perdew ${ }^{1}$, Adrienn Ruzsinszky ${ }^{1}$, Jianwei Sun ${ }^{1}$, Stephen Glindmeyer ${ }^{1}$, and Gabor I. Csonka $^{2}$

${ }^{1}$ Department of Physics and Engineering Physics, Tulane University, New Orleans, LA 70123

${ }^{2}$ Department of Inorganic and Analytical Chemistry, Budapest University of Technology and Economics, H-1521 Budapest, Hungary

\begin{abstract}
The dynamic multipole polarizabilities and thus the second-order van der Waals coefficients $C_{2 k}$ of all orders are known exactly for the interaction between two classical spherical conducting shells, each of uniform electron density $\rho$ with outer radius $R$ and thickness $t$. The result is $C_{2 k}=-c_{k}(t / R) \sqrt{4 \pi \rho}\left[(2 R)^{2}\right]^{k}$. The $c_{k}$ approach a limiting constant value, so the infinite series for the van der Waals interaction at separation $d$, $-C_{6} / d^{6}-C_{8} / d^{8}-\ldots$, can be summed analytically, diverging only for $d \leq 2 R$. This divergence can be removed without changing the asymptotic series. Real quasispherical objects like nanoclusters, fullerenes, and even atoms can be approximated by this spherical-shell model, with $R$ fixed by the true static dipole polarizability. Once $t / R$ is fixed, all the higher coefficients are determined by just $C_{6}$ and $C_{8}$. Finally, we compare the exact $C_{2 k}$ to those from a pair interaction model, which works for solid spheres $(t=R)$ but not for fullerenes.
\end{abstract}

PACS 34.20.Gj, 36.40.-c, 68.65.-k

\section{Introduction to the van der Waals series}

The second-order van der Waals interaction [1] is a long-range attraction between two non-overlapped electron densities, arising from Coulomb correlation between density fluctuations in each. For two spherical objects separated by distance $d$ between their centers, it has the non-retarded infinite asymptotic $(d \rightarrow \infty)$ series

$$
U(d)=-C_{6} / d^{6}-C_{8} / d^{8}-C_{10} / d^{10}-\ldots
$$

For real systems, the van der Waals coefficients are not easy to calculate accurately, especially when the systems are large. Typically, at most only a few leading coefficients are known, with uncertain accuracy. Thus it can be of interest to explore model systems in which these coefficients can be evaluated exactly to all orders, especially when the model systems can approximate real ones of interest. Recently we have examined classical solid-sphere [2, 3] and spherical-shell [4-6] models, which can accurately describe quasispherical nanoclusters, fullerenes, and even atoms, with typical errors of $8 \%$ in the known coefficients. Here we will 
show that, when the two spherical shells are identical, the corresponding van der Waals interaction can be simply summed to all orders, with or without a singularity when the spheres touch. This study should contribute to our understanding of the van der Waals interaction, and perhaps improve the ways in which it is treated in physics, chemistry, and biology, where it provides a dominant mechanism to bind closed-shell systems. In particular, molecular crystals can be built up from fullerenes [7].

The exact Casimir-Polder formula [8] for the van der Waals coefficients between two objects A and B, to second order in the electron-electron interaction, is

$$
C_{2 k}^{A B}=\frac{(2 k-2) !}{2 \pi} \sum_{l_{1}=1}^{k-2} \frac{1}{\left(2 l_{1}\right) !\left(2 l_{2}\right) !} \int_{0}^{\infty} d u \alpha_{l_{1}}^{A}(i u) \alpha_{l_{2}}^{B}(i u),
$$

where $l_{2}=k-l_{1}-1$. Here $\alpha_{l_{1}}^{A}(i u)$ is the $2^{h_{1}}$-pole dynamic polarizability (multipole linear response to an applied uniform electric field) of system A, evaluated at imaginary frequency $\omega=i u: l=1$ (dipole), $l=2$ (quadrupole), $l=3$ (octupole), etc. The infinite subseries of secondorder terms agrees with the full series through order $d^{-10}$ [9] and will be summed here.

\section{Spherical-shell model for the polarizabilities and van der Waals coefficients of all orders}

Consider a classical conducting spherical shell with outer radius $R$, thickness $t$, and uniform density $\rho$ in the region $R-t \leq r \leq R$. We have recently found [4-6] that this is a useful model for quasispherical nanoclusters and atoms $(t=R)$ and for single-walled fullerenes ( $t=2\left(R-R_{n}\right)$, where $R_{n}$ is the radius of the nuclear framework). The radius $R=\alpha_{1}(0)^{1 / 3}$ is found from a realistic value for the static dipole polarizability, which builds in the principal effects of non-metallicity and non-sphericity, and $\rho=N /\left[(4 \pi / 3)\left\{R^{3}-(R-t)^{3}\right\}\right]$ where $N$ is the number of valence electrons. Thus we evaluate Eq. (2) via a controlled interpolation between exact $u=0$ and $u \rightarrow \infty$ limits. The mean absolute relative error of the model $C_{6}$ is about $8 \%$ for cases where accurate reference values are known (atom-atom pairs, $\mathrm{Na}_{\mathrm{n}}-\mathrm{Na}_{\mathrm{n}}, \mathrm{Si}_{\mathrm{n}}-\mathrm{Si}_{\mathrm{n}}, \mathrm{Na}_{\mathrm{n}}-\mathrm{C}_{60}, \mathrm{C}_{60}-\mathrm{C}_{60}$ ) [5]. (Although the free atoms were treated as solid spheres, the exclusion of valence electrons from the core suggests that a hollow-sphere model might be more realistic for them, as it is for the fullerenes. Greater accuracy, around $3 \%$ error for atom pairs, can be achieved by formulas $[2,3$, 5] that make use of the more-realistic radially-varying electron density $\rho(r)$.)

The dynamic multipole polarizabilities for the classical conducting spherical shell are given exactly by Eq. (5) of Lucas et al. [10]. Setting $\varepsilon_{i}=\varepsilon_{e}=1$ and $\varepsilon=1+\omega_{p}^{2} / u^{2}$, where $\omega_{p}=\sqrt{4 \pi \rho}$ is the plasma frequency, we find 


$$
\alpha_{l}(i u)=R^{2 l+1} \frac{\omega_{l}^{2}}{\omega_{l}^{2}+u^{2}} \frac{1-\theta_{l}}{1-\beta_{l} \theta_{l}}
$$

where

$$
\begin{aligned}
& \beta_{l}=\frac{\omega_{l}^{2} \widetilde{\omega}_{l}^{2}}{\left(\omega_{l}^{2}+u^{2}\right)\left(\widetilde{\omega}_{l}^{2}+u^{2}\right)}, \\
& \theta_{l}=\left(\frac{R-t}{R}\right)^{2 l+1}=(1-t / R)^{2 l+1} .
\end{aligned}
$$

Here

$$
\omega_{l}=\omega_{p} \sqrt{l /(2 l+1)}
$$

is the natural frequency of the $l$-th normal mode or surface plasmon in a solid metal sphere, and

$$
\widetilde{\omega}_{l}=\omega_{p} \sqrt{(l+1) /(2 l+1)} .
$$

Note that the static polarizabilities are

$$
\alpha_{l}(0)=R^{2 l+1}(0<t / R \leq 1)
$$

which is remarkably (but correctly for the model) independent of $t$. This occurs for a good classical-physics reason: The equilibrium charge on a conductor is confined to the surface and creates an induced electric field that cancels the applied electric field inside the conductor. The $l=1$ limit is the familiar $\alpha_{1}(0)=R^{3}$. The justification for neglecting the energy gap of the cluster (except insofar as it affects the static dipole polarizability) is given in Ref. [6].

Now the frequency integral in Eq. (2) can be performed, leading to the expressions [5] in Appendix A for the coefficients of all orders. While the model and its dynamic multipole polarizabilities are simple, the corresponding van der Waals coefficients are lengthy analytic expressions which simplify considerably in the solid-sphere limit $t / R \rightarrow 1$ [3], as also shown in Appendix A.

Strictly, our classical model has no zero-point density fluctuation and thus no van der Waals interaction. But it does have exact multipole polarizabilities, which can be and are used in the Casimir-Polder formula of Eq. (2) to generate realistic van der Waals coefficients for quantum systems. This kind of "boot-strapping" also occurs within the adiabatic connection density-functional approach to the random phase approximation (RPA) [11], in which a Hartree density response function (without exchange or correlation) is used to generate an exact exchange energy and an RPA correlation energy. 


\section{Summing the van der Waals series to all orders}

If we set $\mathrm{B}=\mathrm{A}$, we obtain from the Appendix the simple but exact

$$
C_{2 k}=c_{k}(t / R) \sqrt{4 \pi \rho}\left[(2 R)^{2}\right]^{k},
$$

in which all complexity is hidden in the reduced coefficients $c_{k}(t / R)$. The van der Waals series for the interaction energy (Eq. (1)) is then

$$
U(d)=-\sqrt{4 \pi \rho} \sum_{k=3}^{\infty} c_{k}(t / R) z^{k},
$$

where

$$
z=\left(\frac{2 R}{d}\right)^{2}
$$

Some coefficients $c_{k}(t / R)$ for $k \leq 80$ have been computed from the formulas in Appendix A and displayed in Table 1 . They increase modestly from $k=3$, and approach a limiting constant $c_{\infty}$ (apparently independent of $t / R$ ) for large $k$, a behavior that is reminiscent of the geometric series

$$
\sum_{k=1}^{\infty} z^{k}=(1-z)^{-1} \quad(0 \leq z<1)
$$

Thus we can approximately sum the series in a way that will exactly yield the first three terms of Eq. (1), while including the effect of $c_{k} \rightarrow c_{\infty}$ :

$$
U(d)=-\sqrt{4 \pi \rho}\left[c_{3}(t / R) z^{3}+c_{4}(t / R) z^{4}+c_{5}(t / R) z^{5}+c_{\infty}\left\{(1-z)^{-1}-\sum_{k=0}^{5} z^{k}\right\}\right] .
$$

Eq. (13) interpolates between correct large- $d$ and $d \rightarrow 2 R$ limits. That $c_{\infty}$ is independent of $t / R$ is suggested by the fact that $t / R$ enters via $\theta_{l}$, which vanishes as $l \rightarrow \infty$. In Eq. (13), we have assumed that $c_{\infty}$ is indeed independent of $t / R$; in later equations, any dependence of the $c_{k}$ on $t / R$ will be implicit. Figures 1 and 2 compare $U(d)$ to $-C_{6} / d^{6},-C_{6} / d^{6}-C_{8} / d^{8}$, and $-C_{6} / d^{6}-C_{8} / d^{8}-C_{10} / d^{10}$ for the interaction of two solid spheres $\left(\mathrm{Na}_{20}-\mathrm{Na}_{20}\right)$ and for the interaction of two hollow spheres $\left(\mathrm{C}_{60}-\mathrm{C}_{60}\right)$, respectively. Eq. (13) should be most accurate when the $c_{k}$ have the weakest dependence upon $k$, as they do for solid spheres with $t / R=1$. Eq. (13) is consistent with Ref. [12], which shows via inequalities that our Eq. (1) sums to a finite result for nonoverlapped spherical densities $(d>2 R)$. 
The formulas in this section are for the interaction between identical spheres. They are generalized to nonidentical spheres and improved in Eq. (21) below.

\section{Removing the singularity for touching spheres}

Eq. (13) has an unphysical divergence at $z=1(d=2 R$, where the spheres just touch). The divergence is there when we sum up all the terms in Eq. (1), but it is not there in reality because Eq. (1) is only an asymptotic expansion, valid for $d$ so large that the exponential density overlap between the two real quantum-mechanical objects may be neglected. We can remove this singularity, without changing the infinite series to any order in $z$, if we replace $z$ in Eq. (13) by $z^{\prime}=\left(2 R / d^{\prime}\right)^{2}$ where $d^{\prime}$ differs from $d$ by for example a decaying exponential:

$$
d^{\prime}=d+g \times \exp [-(d-2 R) / h]
$$

with $g>0$ and $h>0$ chosen to keep $d^{\prime}>2 R$. Figure 3 shows the result for $g=10$ and $h=2$ atomic units. This choice is arbitrary both in form and in parametrization, and better choices can be made for specific purposes. For example, one might want a long-range correction [13-16] to semilocal density functional approximations (which by themselves provide no long-range van der Waals interaction). Figs. 2 and 3 show that a physical summation is needed for applications.

Similar but stronger singularities at $d=2 R$ seem to be accepted without challenge in the work of Hamaker [17] and Tadmor [18]. These differ from the $d \rightarrow 0$ singularities of the leading terms in Eq. (1), which are conventionally removed via a damping factor [13].

\section{Scaling behavior of the van der Waals coefficients}

By Eq. (9), the van der Waals coefficients of all orders for a pair of identical spherical shells are fixed by the coefficients $\left\{c_{k}(t / R)\right\}$ and by two parameters, $\sqrt{4 \pi \rho}$ and $(2 R)^{2}$ or equivalently $C_{6}$ and $C_{8}$. This means that, if we know the ratio $t / R$ and the first two van der Waals coefficients for a real quasispherical system, we can estimate therefrom the van der Waals coefficients of all orders:

$$
C_{2 k}=C_{6} \frac{c_{k}}{c_{3}}\left[\frac{c_{3} C_{8}}{c_{4} C_{6}}\right]^{k-3} .
$$

An example is a relationship that holds for all spherical shells:

$$
\frac{\left(C_{6} C_{10}\right)^{1 / 2}}{C_{8}}=\frac{\left(c_{3} c_{5}\right)^{1 / 2}}{c_{4}}
$$


The right-hand side of Eq. (16) is 0.9114 for solid spheres $(t / r=1)$ and 0.9065 for hollow spheres with $t / R=0.3403$ (as for $\mathrm{C}_{60}$ ). A similar relationship is known to hold for a simple harmonic oscillator model [19], with 0.9 replaced by 1.1 .

\section{Van der Waals coefficients from Hamaker's pair interaction picture}

In 1937, Hamaker [17] attempted to sum the van der Waals series for the interaction between two solid spheres of uniform density $\rho$, within the pair interaction picture

$$
U(d)=-\beta \int_{A} d^{3} r \int_{B} d^{3} r^{\prime} \frac{1}{\left|\vec{r}{ }^{\prime}-\vec{r}\right|^{6}}
$$

We shall fix the constant pre-factor $\beta$ by requiring that this formula reproduce the exact $C_{6}$ for a pair of identical solid spheres:

$$
\beta=c_{3}(1) \sqrt{4 \pi \rho}\left(\frac{3}{4 \pi}\right)^{2} 2^{6} .
$$

(More generally, for non-identical solid spheres, we can replace $\omega_{p}=\sqrt{4 \pi \rho}$ in Eq. (18) by $2 \omega_{p}^{A} \omega_{p}^{B} /\left(\omega_{p}^{A}+\omega_{p}^{B}\right)$; see Eq. [S5] of Ref. [3].) Hamaker's formula for spheres of equal size (his Eq. (14)) also diverges when (in our notation) $d \rightarrow 2 R$. Tadmor [18] used Hamaker's formula to find a similar interaction between spherical shells. But we note that there is no reason to believe that Eq. (17) is exact. In fact, in other work [6] we have found that an atom pair-potential picture closely related to Eq. (17) can fail badly for fullerenes (hollow spheres). In Appendix B we will extract the van der Waals coefficients $C_{6}, C_{8}$, and $C_{10}$ from the Hamaker and Tadmor (HT) formulas, for comparison with our exact values.

Eqs. (17) and (18) directly imply the simple result

$$
C_{6}^{H T}=-\beta V^{2}
$$

for the interaction of two identical spherical shells of volume V. Since each has a volume $\frac{4 \pi}{3}\left[R^{3}-(R-t)^{3}\right]$, we find

$$
C_{6}^{H T}=-c_{3}^{H T}(t / R) \sqrt{4 \pi \rho}(2 R)^{6}, \quad c_{3}^{H T}(t / R)=0.006766\left[1-(1-t / R)^{3}\right]^{2} .
$$

For solid spheres $(t / R=1)$, the size dependence of $C_{6}^{H T}$ is $R^{6}$ or $n^{2}$, where $n$ is the number of atoms in the cluster. Similarly, as shown in Appendix B, $C_{8}^{H T} \sim R^{8} \sim n^{8 / 3}$ and $C_{10}^{H T} \sim R^{10} \sim n^{10 / 3}$ for solid spheres. 
Taylor expansion of the Hamaker interaction between identical solid spheres and of the Tadmor interaction between identical spherical shells for small $d^{-1}$ yields van der Waals coefficients of the form of Eq. (9) but with modified reduced coefficients $c_{k}(t / R)$ shown in parentheses in Table 1. The Hamaker $c_{3}(t / R=1)$ is exact by our choice of $\beta$, but the Tadmor $c_{3}(t / R<1)$ is seriously too small for single-walled fullerenes with $t / R<<1$. This is consistent with our conclusion of Ref. [6] that the atom pair-potential picture can be correct for solid spheres but not for single-walled fullerenes. Finally, we note that, since the Hamaker van der Waals coefficients have the same $(2 R)^{2 k}$ dependence as our exact treatment, our exact treatment has an asymptotic size dependence [6] for the van der Waals coefficients between identical solid spheres and corresponding quasispherical nanoclusters that is consistent with the atom pairpotential picture. The fullerenes however have a different size-dependence that is inconsistent with this picture [6], because the $t / R$ dependence of the Tadmor $c_{k}$ is incorrect (Table 1).

The problem with the atom pair-potential picture for $C_{6}$ is easy to see: The number of atoms in an object is additive, but the exact dipole polarizability is not. For example, consider adding a spherical carbon cluster at the center of a large fullerene. The static dipole polarizability of the combined system is not the sum of those for the fullerene and for the cluster, but is just the static polarizability of the fullerene by itself, consistent with Eq. (8). A similar problem arises [6] for nonlocal correlation energy functionals of the kind used in Ref. [7]. This kind of radical nonlocality [6] is captured by the random phase approximation or variants thereof [20].

Unlike the less accurate Hamaker-Tadmor $U^{H T}(d)$, our $U(d)$ from Eq. (13) is restricted to the case of identical objects $(B=A)$. We might expect the best of both worlds from

$$
U(d)=-C_{6} / d^{6}-C_{8} / d^{8}-C_{10} / d^{10}+\left(c_{\infty} / c_{\infty}^{H T}\right)\left[U^{H T}(d)+C_{6}^{H T} / d^{6}+C_{8}^{H T} / d^{8}+C_{10}^{H T} / d^{10}\right] .
$$

\section{Summary and conclusions}

In summary, we have found that the van der Waals series of Eq. (1) can be summed as a geometric-like series (Eq. (13) or Eq. (21)). Unlike previous efforts to sum this series [17-19], ours is based on a realistic physical model for the coefficients of all orders, and on an examination (Table 1) of the crucial behavior of the higher-order reduced coefficients. Also unlike previous efforts, we have shown that the resulting unphysical singularity at small non-zero $d$ can be removed without changing the asymptotic series in any order. The higher-order coefficients between identical objects can be estimated from $C_{6}$ and $C_{8}$. The pair interaction model $[17,18]$ produces a series which is qualitatively similar to but quantitatively different from that of the more accurate spherical-shell model. The low-order van der Waals coefficients from the pair interaction model can be reasonably accurate for the interaction between solid spheres, but not between fullerenes. 
Acknowlegments This work was supported in part by the National Science Foundation under Grant No. DMR-0854769 and Cooperative Agreement No. EPS-1003897, with additional support from the Louisiana Board of Regents. We thank J. Tao for foundational work from Ref. [5]. GIC thanks the support by the New Hungary Development Plan (Project ID: TAMOP4.2.2/B-10/1-2010-0009) at BME project.

Appendix A: Detailed expressions of Ref. [5] for the van der Waals coefficients from Eqs. (2) and (3)

$$
\begin{aligned}
& C_{2 k}^{A B}=\frac{(2 k-2) !}{2 \pi} \sum_{l_{1}=1}^{k-2} \frac{R_{A}^{2 l_{1}+1}}{\left(2 l_{1}\right) !} \frac{R_{B}^{2 l_{2}+1}}{\left(2 l_{2}\right) !}\left(\omega_{l_{1}}^{A}\right)^{2}\left(\omega_{l_{2}}^{B}\right)^{2}\left(1-\theta_{l_{1}}^{A}\right)\left(1-\theta_{l_{2}}^{B}\right) I_{l_{1}, l_{2}}^{A B} \\
& I_{l_{1}, l_{2}}^{A B}=\frac{\pi}{2 Q_{l_{1}, l_{2}}^{A B}} \sum_{i=1}^{3}\left[\frac{f_{i}\left(a_{l_{1}}^{A}, a_{l_{2}}^{B} ; b_{l_{1}}^{A}, b_{l_{2}}^{B}\right)}{D\left(a_{l_{1}}^{A}, a_{l_{2}}^{B}\right)}+\frac{f_{i}\left(b_{l_{1}}^{A}, b_{l_{2}}^{B} ; a_{l_{1}}^{A}, a_{l_{2}}^{B}\right)}{D\left(b_{l_{1}}^{A}, b_{l_{2}}^{B}\right)}\right] \\
& Q_{l_{1}, l_{2}}^{A B}=\left[\left(a_{l_{1}}^{A}\right)^{2}-\left(b_{l_{1}}^{A}\right)^{2}\right]\left[\left(a_{l_{1}}^{A}\right)^{2}-\left(b_{l_{2}}^{B}\right)^{2}\right]\left[\left(a_{l_{2}}^{B}\right)^{2}-\left(b_{l_{1}}^{A}\right)^{2}\right]\left[\left(a_{l_{2}}^{B}\right)^{2}-\left(b_{l_{2}}^{B}\right)^{2}\right] \\
& D(x, y)=x y(x+y) \\
& a_{l}=\frac{1}{\sqrt{2}}\left[\left(\omega_{l}^{2}+\widetilde{\omega}_{l}^{2}\right)-\sqrt{\left(\omega_{l}^{2}-\widetilde{\omega}_{l}^{2}\right)^{2}+4 \theta_{l} \omega_{l}^{2} \widetilde{\omega}_{l}^{2}}\right]^{1 / 2} \\
& b_{l}=\frac{1}{\sqrt{2}}\left[\left(\omega_{l}^{2}+\widetilde{\omega}_{l}^{2}\right)+\sqrt{\left(\omega_{l}^{2}-\widetilde{\omega}_{l}^{2}\right)^{2}+4 \theta_{l} \omega_{l}^{2} \widetilde{\omega}_{l}^{2}}\right]^{1 / 2} \\
& f_{1}(p, q ; s, t)=(p q)^{4}+(p q)^{3}\left(s^{2}+t^{2}\right)-p q(s t)^{2}\left(p^{2}+p q+q^{2}\right) \\
& f_{2}(p, q ; s, t)=-\left\{\left(\widetilde{\omega}_{l_{1}}^{A}\right)^{2}+\left(\widetilde{\omega}_{l_{2}}^{B}\right)^{2}\right\}\left[(p q)^{2}\left(p^{2}+p q+q^{2}-s^{2}-t^{2}\right)-p q(s t)^{2}\right. \\
& f_{3}(p, q ; s, t)=\left(\widetilde{\omega}_{l_{1}}^{A}\right)^{2}\left(\widetilde{\omega}_{l_{2}}^{B}\right)^{2}\left[p^{4}+p^{3} q+p^{2} q^{2}+p q^{3}+q^{4}\right. \\
& \left.-\left(p^{2}+p q+q^{2}\right)\left(s^{2}+t^{2}\right)+(s t)^{2}\right]
\end{aligned}
$$

These complicated formulas simplify considerably for identical $(\mathrm{B}=\mathrm{A})$ solid $(t=R)$ spheres:

$$
C_{2 k}^{A A}=\omega_{p}(2 R)^{2 k} \frac{1}{2^{2 k}} \frac{(2 k-2) !}{4} \sum_{l=1}^{k-2} \frac{1}{(2 l) !(2 k-2 l-2) !} \frac{1}{\sqrt{(2 l+1) / l}+\sqrt{(2 k-2 l-1) /(k-l-1)}}
$$




\section{Appendix B: Van der Waals coefficients in the Hamaker -Tadmor pair-interaction picture}

In the Hamaker pair-interaction picture of Eq. (17), the van der Waals interaction between two solid spheres of radii $R_{A}$ and $R_{B}$, with separation $d$ between their centers, is [17]

$$
U^{H}\left(R_{A}, R_{B}, d\right)=\left(-\pi^{2} \beta / 6\right)\left\{\frac{2 R_{A} R_{B}}{d^{2}-\left(R_{A}+R_{B}\right)^{2}}+\frac{2 R_{A} R_{B}}{d^{2}-\left(R_{A}-R_{B}\right)^{2}}+\ln \left[\frac{d^{2}-\left(R_{A}+R_{B}\right)^{2}}{d^{2}-\left(R_{A}-R_{B}\right)^{2}}\right]\right\}
$$

where $\beta$ is defined below Eq. (18). The Taylor expansion of Eq. (B1) in powers of $d^{-1}$ is

$$
-\frac{\pi^{2} \beta}{6}\left(\frac{32 R_{A}^{3} R_{B}^{3}}{3 d^{6}}+\frac{32 R_{A}^{3} R_{B}^{3}\left(R_{A}^{2}+R_{B}^{2}\right)}{d^{8}}+\frac{64\left(5 R_{A}^{7} R_{B}^{3}+14 R_{A}^{5} R_{B}^{5}+5 R_{A}^{3} R_{B}^{7}\right)}{5 d^{10}}\right)+\ldots
$$

For two identical spheres (A=B), Eq. (B2) becomes

$$
-\frac{\pi^{2} \beta}{6}\left(\frac{32 R_{A}^{6}}{3 d^{6}}+\frac{64 R_{A}^{8}}{d^{8}}+\frac{1536 R_{A}^{10}}{5 d^{10}}\right)+\ldots
$$

Tadmor [18] started from Hamaker's formula (B1), then imagined each sphere as the sum of a spherical shell and a solid-sphere core filling the interior of the shell, so that

$$
\begin{aligned}
U^{H T}\left(\text { shell }_{A}, \text { shell }_{B}\right) & =U^{H}\left(\text { sphere }_{A}, \text { sphere }_{B}\right)-U^{H}\left(\text { sphere }_{A} \text { core }_{B}\right)-U^{H}\left(\text { core }_{A}, \text { sphere }_{B}\right) \\
& +U^{H}\left(\text { core }_{A}, \text { core }_{B}\right)
\end{aligned}
$$




\section{References}

[1] A.J. Stone, The Theory of Intermolecular Forces (Clarendon Press, Oxford, 1996).

[2] J. Tao, J.P. Perdew, and A. Ruzsinszky, Phys. Rev. B 81, 233102 (2010).

[3] J. Tao, J.P. Perdew, and A. Ruzsinszky, Proc. Nat. Acad. Sci. (USA) 109, 18 (2012).

[4] J.P. Perdew, J. Tao, P. Hao, A. Ruzsinszky, G.I. Csonka, and J.M. Pitarke, J. Phys.: Condens. Matter 24, 424207 (2012).

[5] J. Tao and J.P. Perdew, submitted.

[6] A. Ruzsinszky, J.P. Perdew, J. Tao, G.I. Csonka, and J.M. Pitarke, Phys. Rev. Lett. 109, 233203 (2012).

[7] K. Berland, O. Borck, and P. Hyldgaard, Computer Phys. Commun. 182, 1800 (2011).

[8] H.B.G. Casimir and D. Polder, Phys. Rev. 73, 360 (1948); S.H. Patil and K.T. Tang, J. Chem. Phys. 106, 2298 (1997).

[9] V.D. Ovsiannikov and J. Mitroy, J. Phys. B: At. Mol. Opt. Phys. 39, 159 (2006).

[10] A.A. Lucas, L. Henrard, and P. Lambin, Phys. Rev. B 49, 2888 (1994).

[11] D.C. Langreth and J.P. Perdew, Phys. Rev. 21, 5469 (1980).

[12] Y.-H. Zhang, L.-Y. Tang, X.-Z. Zhang, J. Jiang, and J. Mitroy, J. Chem. Phys. 136, 174107 (2012).

[13] Q. Wu and W. Yang, J. Chem. Phys. 116, 515 (2002).

[14] E.R. Johnson and A.D. Becke, J. Chem, Phys. 123, 024101 (2005).

[15] A. Tkatchenko and M. Scheffler, Phys. Rev. Lett. 102, 073005 (2009).

[16] S. Grimme, Wiley Reviews - Computational Molecular Science 1, 211 (2011).

[17] H.C. Hamaker, Physica 4, 1058 (1937).

[18] R. Tadmor, J. Phys.: Condens. Matter 13, L195 (2001).

[19] A.J. Thakkar and V.H. Smith, J. Phys. B 7, L321 (1974).

[20] W.M. Zhu, J. Toulouse, A. Savin, and J.G. Angyan, J. Chem. Phys. 132, 244108 (2010).

[21] P.A. Heiney, J.E. Fischer, A.R. McGhie, W.J. Romanow, A.M. Denenstein, J.P. McCauley, and A.B. Smith, Phys. Rev. Lett. 66, 2911(1991). 
Table 1 Reduced van der Waals coefficients of Eq. (9), for classical solid spheres $(t / R=1)$ and hollow spheres $\left(t / R=0.3403\right.$ as for $\mathrm{C}_{60}$, and 0.0531 as for $\left.\mathrm{C}_{3840}\right)$. In parentheses are values predicted by the pair interaction model of Eqs. (17) and (18), via our Taylor expansion of expressions by Hamaker [17] for $t / R=1$ and by Tadmor [18] for $t / R<1$. The HamakerTadmor value for $c_{\infty}$ is derived from Eq. (14a) of Ref. [17].

\begin{tabular}{llll}
\hline Coefficient & $t / R=1$ & $t / R=0.3403$ & $t / R=0.0531$ \\
\hline$c_{3}$ & 0.006766 & 0.005448 & 0.002237 \\
& $(0.006766)$ & $(0.003437)$ & $(0.000154)$ \\
$c_{4}$ & 0.008842 & 0.007547 & 0.003221 \\
& $(0.01015)$ & $(0.006328)$ & $(0.000366))$ \\
$c_{5}$ & 0.009599 & 0.008592 & 0.003835 \\
& $(0.01217)$ & $(0.008857)$ & $(0.000649)$ \\
$c_{6}$ & 0.009946 & 0.009215 & 0.004313 \\
$c_{10}$ & 0.010447 & 0.010273 & 0.005753 \\
$c_{20}$ & 0.010761 & 0.010757 & 0.007988 \\
$c_{40}$ & 0.010904 & 0.010908 & 0.009960 \\
$c_{80}$ & 0.010979 & 0.010979 & 0.010870 \\
$c_{\infty}$ & 0.011 & 0.011 & 0.011 \\
& $(0.020)$ & $(0.020)$ & $(0.020)$
\end{tabular}




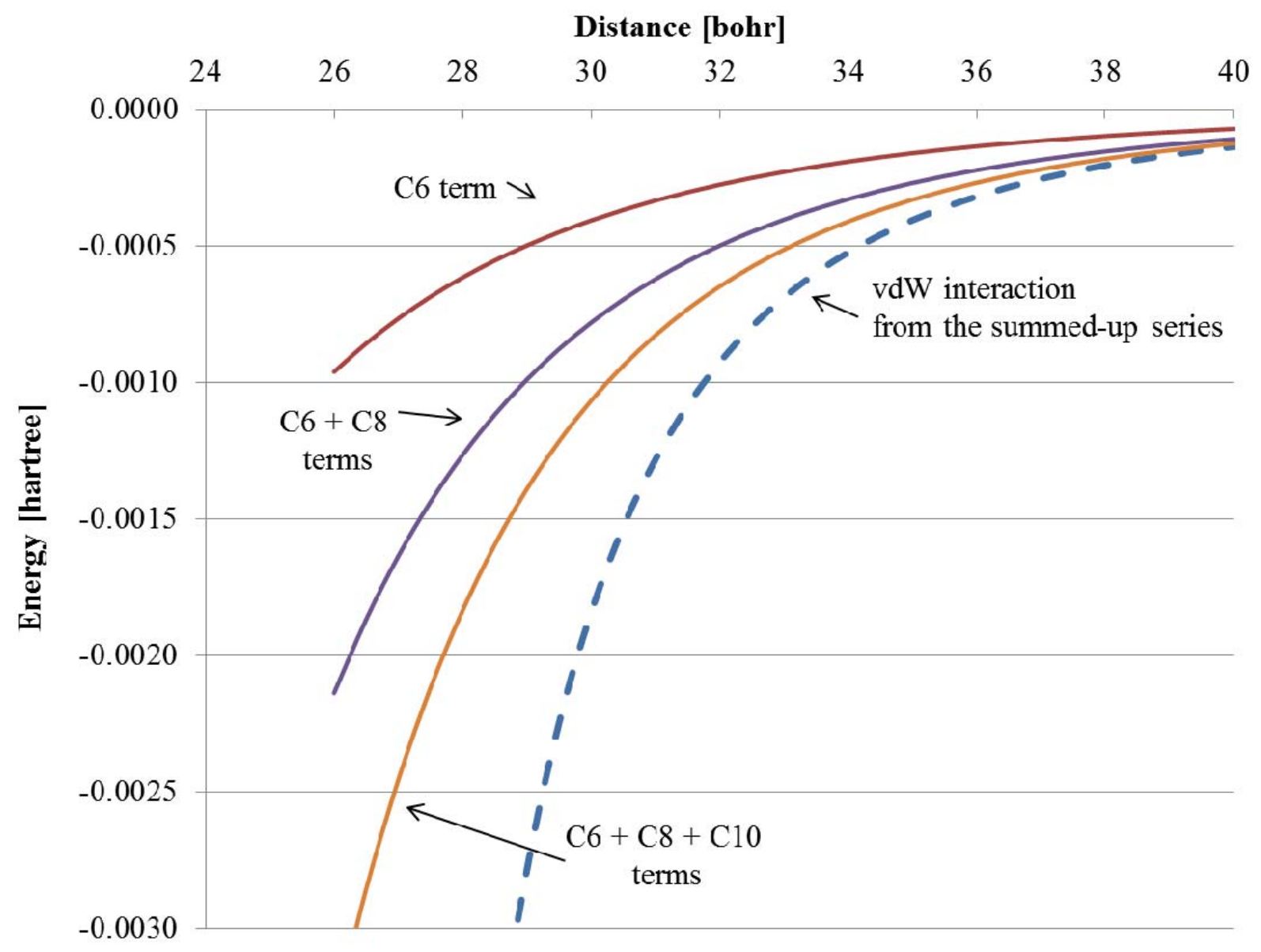

Figure 1: (Color online) Binding energy curves for the nanocluster pair $\mathrm{Na}_{20}-\mathrm{Na}_{20}$, with the multipole polarizabilities of each $\mathrm{Na}_{20}$ modeled as those of a classical conducting solid sphere with $R=12.57$ bohr, $t=R$, and $N=20$. The curves show the leading contributions to Eq. (1), and also Eq. (13) which sums the asymptotic series of Eq. (1) to all orders. 


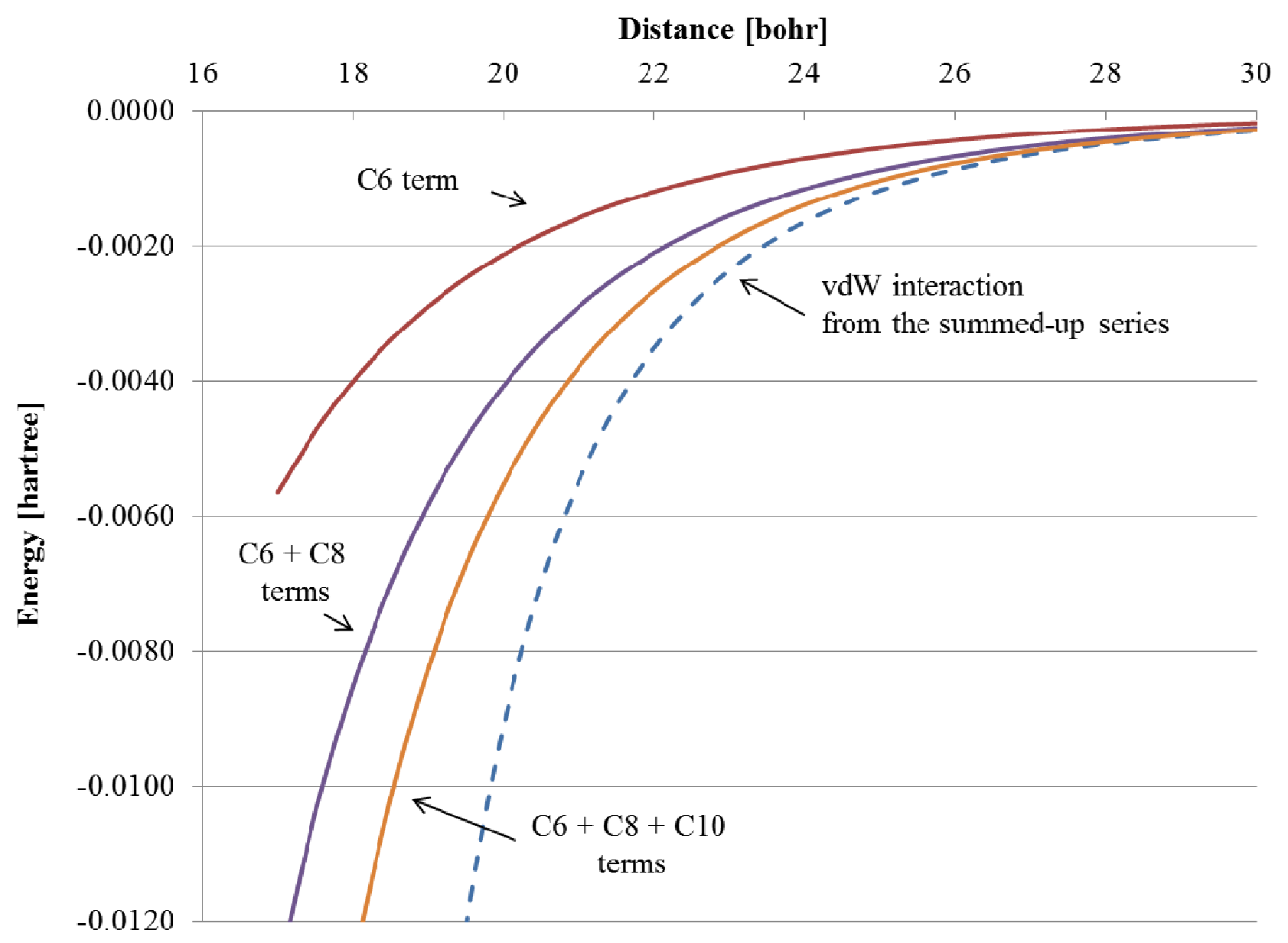

Figure 2: (Color online) Binding energy curves for the fullerene pair $\mathrm{C}_{60}-\mathrm{C}_{60}$, with the multipole polarizabilities of $\mathrm{C}_{60}$ modeled as those of a classical conducting spherical shell with $R=8.11$ bohr, $t=2.76$ bohr, and $N=240$. The curves show the leading contributions to Eq. (1), and also Eq. (13), which sums the asymptotic series of Eq. (1) to all orders. 


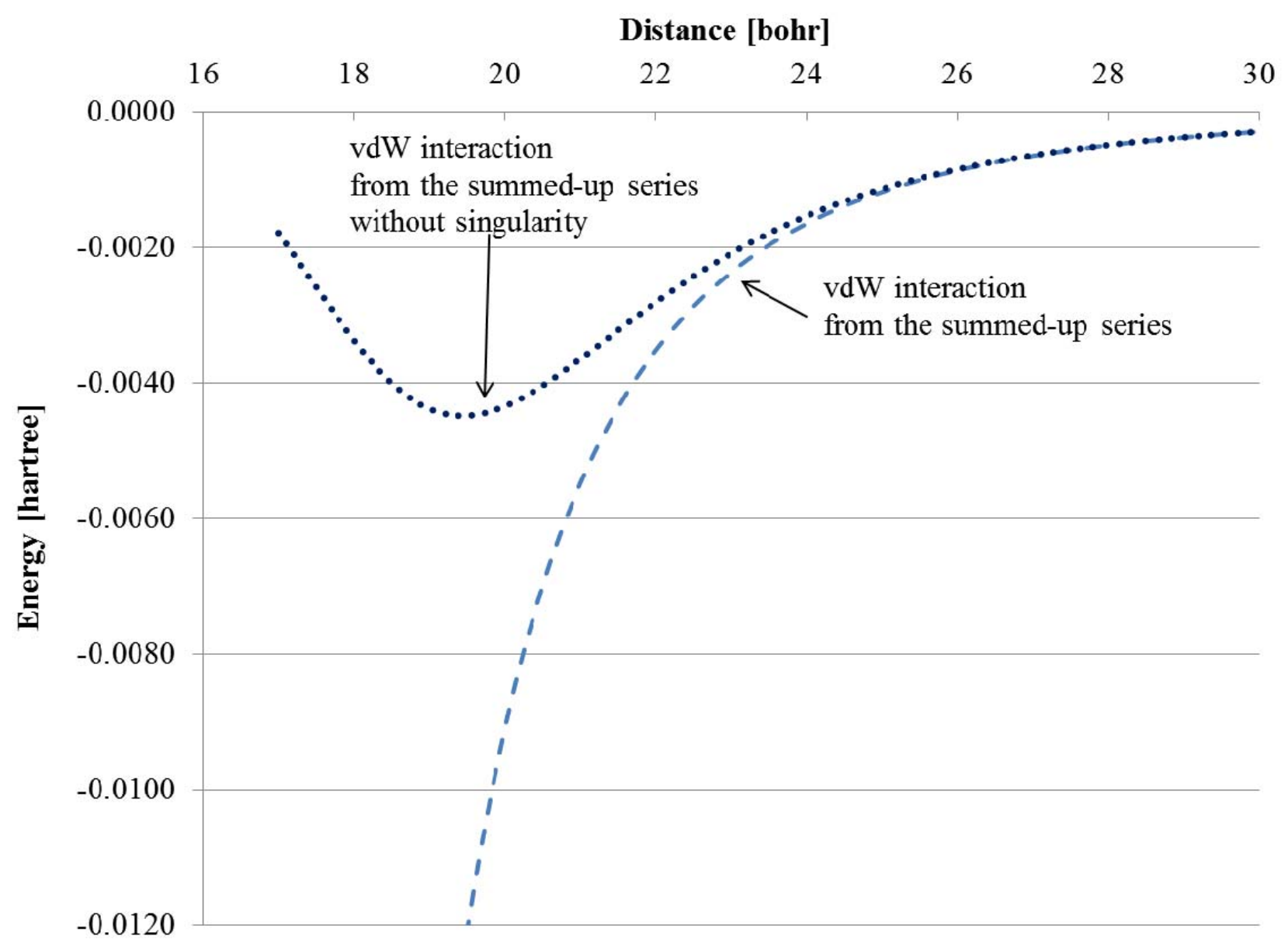

Figure 3 (Color online) Binding energy curves for $\mathrm{C}_{60}-\mathrm{C}_{60}$. van der Waals interaction summed to all orders with and without a singularity at $d=2 R$. The curves show Eq. (13), which sums the asymptotic series of Eq. (1) to all orders, and Eq. (14) (with $g=10$ and $h=2$ ), which has the same asymptotic $\quad(d \rightarrow \infty)$ expansion without the singularity at $d=2 R$. (The equilibrium separation between the centers of neighboring buckyballs in the orientationally-disordered facecentered-cubic molecular crystal is 18.9 bohr [21].) 